points of ophthalmology which are found in Morgagni's various works.

In the third section he deals critically with each point, and shows how our modern knowledge is connected with that of Morgagni.

Morgagni's investigations covered eleven parts of the field; not only anatomy and physiology, human and comparative, but also pathology interested him, and he may be called the founder of pathological anatomy. He referred all questions of anatomy to the best authority, the examination of the body itself, and thus he was able to correct many erroneous ideas which were current.

The marshalling of the facts in the form that Ovio has chosen, will carry conviction concerning his thesis, to every impartial reader. But the form is somewhat dry, and this will limit the number of those who will become familiar with the book.

HaRold Grimsdale.

Optotypes consisting of Test-letters and Pictographs for measuring the Acuteness of Vision. By DR. JOHN GREEN AND DR. A. E. EwIng. London: Henry Kimpton. Thirtyfive engraved plates and an explanatory pamphlet of 24 pages. Price 25s. 1923.

The foreword and first two sections of the pamphlet were written by the late Dr. Green previous to his death in 1913. Two hundred copies of the tests were published at the same time but without the book. These copies were given to personal friends. Dr. Ewing has now completed the book and reprinted the tests, the letters from the original plates of 1886 and the pictographs from the plates of 1902. The pamphlet explains the mathematical basis on which the size of the letters and pictographs as well as the distance at which they should be read have been calculated. The test cards are well reproduced, and should be found of considerable use in the accurate noting of subjective

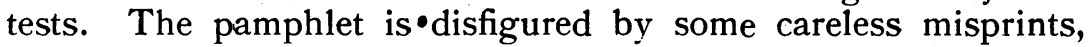
one of which occurs even on the cover.

\title{
CORRESPONDENCE
}

\section{SEPSIS IN RELATION TO GLAUCOMA}

To the Editor of The BRItish JouRnal of Ophthalmology

SIR,-Perusal of the literature of glaucoma affords so many facts suggesting a close association between this disease and sepsis that a more thorough examination of the question is indicated. 
Recently the writer examined five cases of glaucoma and in every instance, culture from the conjunctiva gave a heavy growth of staphylococcus albus. Pieces of uvea from four cases were incubated, and two of them, from cases of relatively recent glaucoma gave a growth of staphylococcus albus, while two from cases of chronic glaucoma showed no growth.

There are reasons for thinking that the growths obtained from the uvea were not due to contaminations and that the germ was actually present in the tissues.

In taking cultures from the conjunctiva the spatula was carried well below the surface of the agar, and in every instance there was heavy growth extending from the surface of the medium to the end of the track made by the stab.

In incubating the uvea, the tissue was carried well below the surface of the medium with the spatula, and in no case was there infection of the surface or of the track left by the stab, so that evidence of contamination in the uveal cultures is not strong.

In two recent cases there was a fairly vigorous growth from the tissues outwards, and in two chronic cases there was no growth.

That the chronic cases should show no growth does not in any way contra-indicate a septic origin of the disease. During a septic attack the germ becomes less virulent, and it is not unusual, for instance, in chronic pus tubes, to find that the infective agent cannot be demonstrated, but there is no question as to the infective origin of the disease.

In chronic glaucoma the vascular degeneration is such that we should expect progressive atrophy in the tissues, even if the germ were no longer viable and there were no question of toxic absorption. I was much struck recently when eviscerating an eyeball in a case of fairly recent glaucoma by the fact that the haemorrhage seemed to be purely arterial, whereas in a case done a short time previously for severe trauma the haemorrhage appeared to be purely venous.

The cultures from the uvea, in the cases which gave positive results, were so different in character and appearances from the conjunctival cultures as to give the impression that they were due to a different germ, until the microscope proved that, morphologically at least, they were the same. As the staphylococcus to become viable in the uvea must, to some extent at least, take on an anaerobic habit of life, its characteristics must be greatly modified and cultures from it would also show great differences from those of the aerobic germ.

As there is so marked a difference between the conjunctival and the uveal cultures in these cases the matter demands investigation, and I am drawing attention to it because this method of attacking the problem has not been generally used, and because it is to be hoped 
that others will take up the investigation as its solution seems to depend upon a multiplicity of observation.

If we find in our cases of recent glaucoma, cultures of staphylococci from the uvea, which differ consistently from those obtained from the conjunctiva, we cannot, a priori, exclude the staphylococcus as a potent factor in the disease.

Montreal.

Yours truly,

RICHARD KERRY.

\section{THE LARGE LENS OF THE GULLSTRAND SLIT-LAMP}

\section{To the Editor of The British Journal of Ophthalmology}

SIR,-Those familiar with the slit-lamp will be aware that only an elongated vertically disposed central portion of the lens referred to above is made use of, the portions of the lens on either side of this being superfluous. The area used is defined by the aperture in the diaphragm, which elsewhere covers the superfluous portions of the lens. The lens is of large diameter $(50 \mathrm{~mm}$.) in order that it may receive rays, emerging from the slit-diaphragm, covering a relatively wide vertical angle. The lens being accurately corrected, and being of such large diameter, produces a good intensity of focal illumination. Even so, the intensity with the ordinary Nernst-filament slit-lamp leaves no spare power to waste; for high power work, the intensity with this lamp sets many limitations.

The large cross-diameter of the lens is undesirable, as most owners of the apparatus must have experienced, because it prevents the illuminator being brought to a sufficiently acute angle with the objectives of the microscope to enable the observer thus to view the deeper parts of the vitreous, only the vitreous just behind the lens and not that which is deeper being directly accessible to view. The present method of looking deeper into the vitreous is to make an elaborate readjustment of the instrument and employ Koeppe's reflecting mirror to procure the deeper view. This mirror is a very necessary accessory for certain procedures, and familiarity with its use is called for; but when time is limited it is a disadvantage to be compelled to make a gross readjustment of the apparatus every time that a rather deeper glance into the vitreous is desired.

There are, moreover, other instances, in routine observation of more anterior parts of the eye, in which facility for a simple change to an acute angle between the axes of illumination and observation is desirable. This is rendered feasible by Dr. Vogt's "small 